\title{
Numerical Simulation and Experimental Study of Electrostatic Field near Man with Protective Polymeric Clothing
}

\author{
Larisa Tretiakova ${ }^{1, *}$, Alexander Podoltsev², Ostapenko Natalia ${ }^{3}$ \\ ${ }^{1}$ National Technical University of Ukraine «Kyiv Polytechnic Institute», Ukraine \\ ${ }^{2}$ Institute of Electrodynamics of NAS of Ukraine, Ukraine \\ ${ }^{3}$ Department of Ergonomics and Design Clothes, Kiev National University of Technology and Design, Ukraine
}

Copyright $\subseteq 2017$ by authors, all rights reserved. Authors agree that this article remains permanently open access under the terms of the Creative Commons Attribution License 4.0 International License

\begin{abstract}
In the article the mathematical model and the method of numerical calculation, based on the method of finite elements of three-dimensional electrostatic field near-by a man at presence of surface charge on his protective clothing, made from polymeric material, were considered. Experimental researches were conducted to determine the value of the surface charge. The results of numeral calculations of the distribution of electric potential and field intensity, as well as integral value of electric energy, stored in this field, were presented.
\end{abstract}

Keywords Electrostatic Field, Protective Clothing of Workers, Simulation, Results of Numeral Calculations

\section{Introduction}

Modern industrial production raises high demands to the technical improvement of equipment, processes and systems of protection against the negative impact of harmful factors. An important role in the implementation of multilevel system of workers' protection belongs to the personal protective equipment (PPE), which make it possible to reduce the negative impact of the physical, chemical and biological factors and harmful substances. Today, for the production of PPE (insulating and air-tight clothes, respirators and gas masks, tools for arms, legs, head and eyes protection) polymeric materials with the volume resistance within $10^{9} \ldots 10^{16}(\Omega \cdot \mathrm{m})$ are widely used. Such materials provide an effective protection against the numbers of harmful factors, but in properties they are dielectrics and create additional risks in the form of an electrostatic field (ESF) on the outer and inner layers of the surfaces of protective equipment. Such products can accumulate and store charges for a long period of time and form ESF with a certain level of intensity and energy.

The aim of the article is to develop the mathematical model and method of ESF calculation on the surface of the worker's protective clothing, made fr2om polymeric material, considering the available information about the charge distribution.

\section{Materials and Methods}

On the surfaces of the protective clothing ESF form during the process of contact electrification, which takes place during the collision of two surfaces with the different dielectric constants: surface material of PPE with other dielectric or air flow; between internal heterogeneous layers of clothing, underwear and body of the worker. Another way of formation of static charges is induction, which occurs when the worker stands near the strong external ESF. The greatest danger comes from the human body that can accumulate static charges and create huge electrostatic potentials. Protective clothing together with gloves, helmet, respirator, protective footwear that has dielectric sole is a good dielectric; this conduces to the accumulation of electric charges on the human body. When workers do their work in such protective clothing sets, ESF with potentials forms at the surfaces from 8 to $25 \mathrm{kV}$ and at the human bodies $-2 \ldots 8$ $\mathrm{kV}[1]$.

Inside the energy providers buildings electronic means of control and measurement are installed everywhere, which are especially sensitive to the impacts of hindrances, created by external ESF. Despite the fact that the electrostatic charge does not contain large amount of energy, high difference in potentials contributes to the formation of currents, sufficient both for the instantaneous malfunction of sensitive electronic components and for inner molecular damage of their crystal lattices. Damage or malfunction of electronic devices at the workplaces, panels of control, in control room can occur 
because of the discharges of static electricity from the worker's protective clothing set, as evidenced by numerous studies [2]. Potential, connected to the electrostatic charge, can erase coded information from the computer memory unit and create barriers to the electronic communications. For most of microelectronic products static discharge $5 \ldots 10 \mathrm{kV}$ is devastating. Researches, made on energy facilities, found that the major part of $50 \%$ possible extra voltages, which had resulted in damages of electronic devices for diagnostics, management, storage and transfer of information or their improper operation, was connected with ESF that had formed on the protective equipment - on the clothes, gloves, respirators [3].

The unique feature of ESF is a creation of the conditions for the formation of self-discharge and electric spark (interval breakdown), as well as strong enough ionization of the environment that leads to the negative impact on the worker's health and to the potential risk of injuries. Most people feel uncomfortable in the presence of voltage $1.5 \ldots 2$ $\mathrm{kV}$; individual organs and tissues in the human body are sensitive to the potentials in the range $100 \ldots 150 \mathrm{~V}$ [4].

Electrical discharge may be sufficient to ignite flammable or explosive mixture or fibers. For example, for some steam and gas-air explosive mixtures a small energy, ranged from $0.1 \cdot \mathrm{MJ}$ is needed, for dust and fibers - ranged from $100 \mathrm{~mJ}$ is needed as well. Such risks, which arise from static electricity, are limited in regulatory documents on labor protection [5]. At the production spaces it should be prevented the occurrence of spark discharges from static electricity with the energy that exceeds $40 \%$ of the minimum energy of spark discharge in the environment. Almost at the voltage of 5.5 $\mathrm{kV}$, the energy of spark reaches $1.1 \mathrm{~mJ}$, which is sufficient to ignite steam and gas-air explosive mixtures, and the most of flammable dust and fibers at the voltage of $10 \mathrm{kV}$. Every year the accidents are recorded caused by electrostatic discharges from the surfaces of PPE, which are accompanied by major accidents and material losses.

Thus, ESF, which is formed on the surfaces of the protective clothing sets, leads to the following negative consequences:

- harmful effect of ESF and micro discharges from the surface of PPE, which occur in such field, on the worker's health;

- formation of ESF with high potentials that has an impact on the electronic and computer control systems; - occurrence of discharges and additional electromagnetic fields that creates a risk of explosion or fire.

Static electricity is a surface phenomenon; that's why its appearance or disappearance depends on the surface resistance of material, which becomes electrified. In the effective standards the value of electrical resistance is normed for the products, made from polymeric materials [6]. The methods of resistance evaluation are well developed, do not require expensive instrumentation and let you to get single-valued results under normal conditions $\left(t=20{ }^{\circ} \mathrm{C}\right.$, humidity $\mathrm{W}=60 \%$ ). However, the value of surface resistance is influenced by several factors. It is determined for the materials from polyvinyl chloride (PVC) that their properties depend on the mode and method of manufacturing, on the structure of materials and composition of raw materials with stabilizers, modifiers, plasticizers, antistatic and colorants. Thus, the addition of modifiers from oxides of rare earth elements to the composition of PVC-compound reduces the resistance from $2.1 \cdot 10^{13}$ to $3.17 \cdot 10^{11} \Omega$. A number of external factors significantly affect as well, including the most important ones: temperature, humidity, radiation, the presence of aerosol pollutions [7]. Therefore, to study the influence of ESF, it is not enough to use only one parameter - resistance; it is necessary to know its main characteristics: intensity, distribution of charges and potentials, energy.

To measure the quantitative parameters of ESF, series of field tests at nuclear power plants were made. At nuclear power plants of Ukraine workers used protective clothes, made from materials, characteristics of which are given in Table $1[8]$.

Table 1. Electrical resistance of materials of protective clothing

\begin{tabular}{|c|c|c|c|c|}
\hline Type of material & $\begin{array}{c}\text { Coded identification of } \\
\text { material }\end{array}$ & Thickness, mm & Humidity $60 \%$ & Humidity $100 \%$ \\
\cline { 4 - 5 } & V & 0.12 & $2.11 \cdot 10^{13}$ & $5.68 \cdot 10^{9}$ \\
\hline $\begin{array}{c}\text { PVC-compound without antistatic } \\
\text { agents }\end{array}$ & RP1 & 0.52 & $1.43 \cdot 10^{13}$ & $2.35 \cdot 10^{11}$ \\
\hline $\begin{array}{c}\text { PVC-compound with modifier - silicon } \\
\text { wolframite }\end{array}$ & RP2 & 0.55 & $3.17 \cdot 10^{11}$ & $6.51 \cdot 10^{9}$ \\
\hline $\begin{array}{c}\text { PVC-compound with modifier of a } \\
\text { mixture }\end{array}$ & IB & 0.3 & $7.92 \cdot 10^{13}$ & $1.62 \cdot 10^{10}$ \\
\hline Ofwo-layer material with PVC covering & PE & 0.12 & $5.16 \cdot 10^{9}$ & $1.32 \cdot 10^{9}$ \\
\hline Polyethylene 'Tajvek" & PP & 0.47 & $4.32 \cdot 10^{11}$ & $3.82 \cdot 10^{9}$ \\
\hline Polypropylene "Xiмволокн" & F & 1.82 & $1.64 \cdot 10^{8}$ & $2.62 \cdot 10^{3}$ \\
\hline $\begin{array}{c}\text { Composite textile material } \\
\text { "KÄRCHER" }\end{array}$ & & & \\
\hline
\end{tabular}


The measurement of ESF parameters was made using the device ВПЕП-1. The device enables to measure potentials and charges in the range: surface charge density $-10^{-12} \ldots 10^{-5}$ $\mathrm{C} / \mathrm{m}^{2}$; intensity $-20 \ldots 2 \cdot 10^{6} \mathrm{~V} / \mathrm{m}$; potentials $-0.02 \ldots 5 \cdot 10^{3} \mathrm{~V}$.

The density of the charges essentially depends on the conditions in which the electrification occurs, including the type, condition and surface area of the contacting materials, the presence of microinclusions in the form of water, dust. It was found during the researches that in the process of work the surface density changed on the surface of the set, as well as through time in the range from $2 \cdot 10^{-13}$ till $1 \cdot 10^{-7} \mathrm{C} / \mathrm{m}^{2}$. The highest density of charges was documented at the surfaces of the insulating protective clothing, shoes, made of PVC-compound, and at full gas masks. The lowest density was documented at the overalls, made from polyethylene brand "Tajvek", which had the lowest surface resistance and corrugated surface. It was found that charges distributed unevenly on the surfaces of the protective equipment: the highest density of charges was documented on the body in the chest area, in front of clothing and on the head of the worker, the lowest - on the legs and arms. The phenomena that occur become more complicated because of the different types of surface contamination and internal impurities.

The process of accumulation and drainage of charges from the surfaces is quite fleeting. More often the discharge effect occurs for workers at the time of stripping of protective clothing.

When humidity increase, the decrease of the maximum values of the charge on the surface of clothes was registered (Fig. 1.) accompanied by the formation of microinclusions in the form of water drops

Charge density, $10^{-7} \mathrm{C} / \mathrm{m}^{2}$

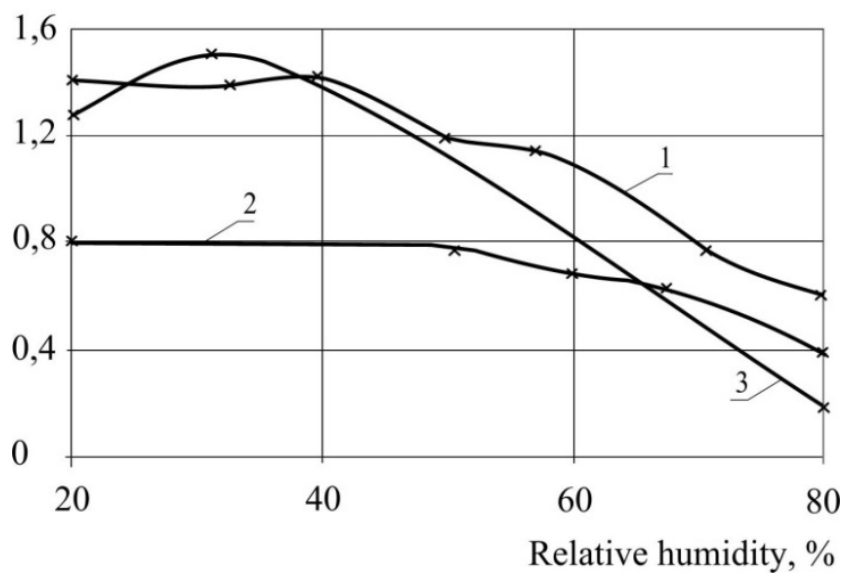

Figure 1. Dependence of the maximum surface density of charges from the humidity on the surface of protective clothing, made from the materials with coded identification: 1 - PP; 2 - PE; 3 - V.

Field measurements of potentials (Fig. 2), which are formed on the surfaces of protective clothing, showed that they are in range $3000 \ldots 8000 \mathrm{~V}$.
Potential, kV

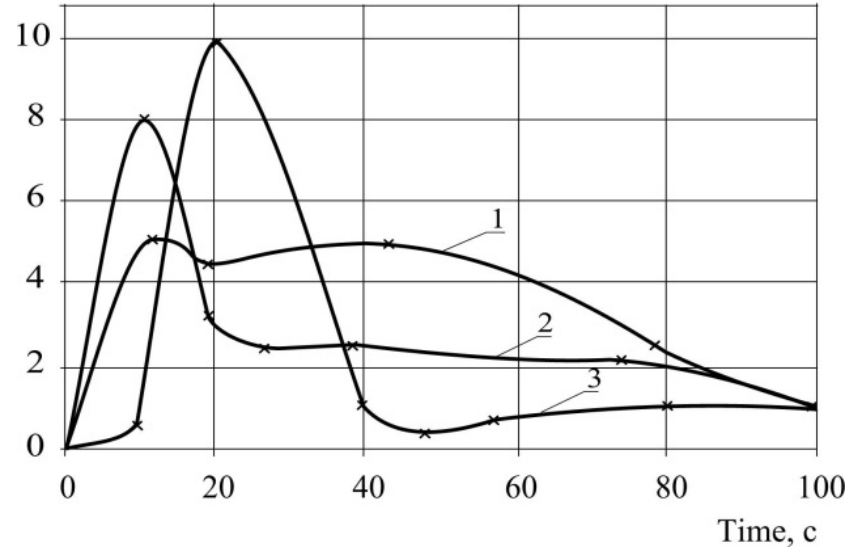

Figure 2. Dependence of the potential from the time on the outside surfaces of insulating protective clothing made from the following materials: $1-\mathrm{IB} ; 2-\mathrm{V} ; 3-\mathrm{PE}$

The maximum value of potentials was registered as follows: protective clothing made of PVC-compound - up to $10 \mathrm{kV}$; on the outside surfaces of gas masks, brand MP - up to $7 \mathrm{kV}$; on the gloves - up to $5 \mathrm{kV}$.

Field experiments have shown that ESF on the surface of the protective clothing set is characterized by the high degree of heterogeneity and uncertainty: there is uneven distribution of charges on the areas of clothing; charges distribute on the outer and inner surfaces of various sizes and characters; there is a constant drip of charges, caused by conductivity, wetting and pollution. In addition, significantly affect the environment parameters, material properties, design and geometric dimensions of protective equipment. Obtainment of complete information about ESF parameters is connected with great time-consuming work. Therefore, in practice, researchers are limited to single random results of measurements, on which they do analysis and make decisions that can lead to accidents.

Analysis and evaluation of ESF parameters, especially risks for workers and electronic equipment, need the development of the method of mathematical simulation, which provides an opportunity to identify the level of ESF energy indicator, on which normal interaction of all elements of concerned system is guaranteed with the highest level of possibility.

It should be noted that the analysis of the surface ESF in heterogeneous dielectric environment is a difficult task. The solution to this problem with the use of analytical approach in final form is possible only for certain simplified cases, limited by certain conditions: by rectilinear surfaces of infinite length; by homogeneous environment with equal surface charge. Reduction of the real three-dimensional ESF to such conditions on the curved surfaces with microinclusions and with variable density of charges, which occurs on the surfaces of protective clothing, is not possible. 


\section{Results}

The summary of test method is in the purposeful change of density of electric charge in time, as well as in some areas of protective elements. The ultimate aim of the calculation is the quantitative determination of the ESF values distribution and their limit values, which satisfy a number of limitations: limits of the ignition energy, limits of the acquired surface potential and limits of charges drainage speed.

The calculation of three-dimensional ESF, generated near the worker in protective clothing during the accumulation of surface charges on it, made in these terms:

1. We create a geometric model of human, combining various basic geometric shapes.

2. In the model it is provided that the entire surface of the worker's clothing is covered with the polymeric materials with thickness $0.1 \ldots 0.5 \mathrm{~mm}$ that corresponds to the actual thickness of the used materials: for the protective clothing and gloves the thickness of material is $0.1 \ldots 0.2 \mathrm{~mm}$; for respirators, gas masks, hoods and shoes $-0.3 \ldots 0.5 \mathrm{~mm}$. As a result of friction or induction impact, surface charge with density of $\sigma_{q}(x, y, z)$ formed on the surface of the material. According to the results of field observations, it was provided that surface charge density varied according to the area $S$ of the product.

3. Estimated section is a section of the air environment, external to the human; the boundary condition for the normal component of ESF intension is specified for the surface of the human body. Materials, which contact, and microinhomogeneities are dielectrics [7] with relevant electrical characteristics. Distribution of constant over time ESF near-by a man can be described by the following differential equation for the scalar electric potential [2]:

$$
\operatorname{div}\left(-\varepsilon_{0} \operatorname{grad} \varphi\right)=0,
$$

where $8.85 \cdot 10^{-12} \mathrm{~F} / \mathrm{m}$ - constant permittivity of vacuum.

Boundary condition on the surface is as follows:

$$
-\left.\varepsilon_{0} \frac{\partial \varphi}{\partial n}\right|_{S}=\sigma_{z}
$$

To circuit the calculated area, the model of human was placed into a cube with the size of rib $4 \mathrm{~m}$, while its lower face considered grounded, and for the top and side faces of the cube was established the condition of electrical insulation, which has the form (see Fig. 3):

$$
-\left.\varepsilon_{0} \frac{\partial \varphi}{\partial n}\right|_{\Gamma 2-6}=0
$$

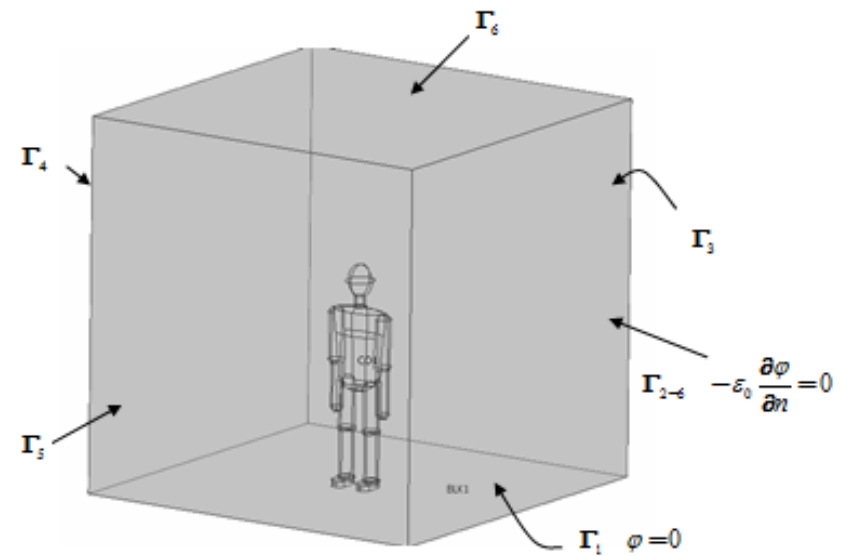

Figure 3. The calculated areas for the finite-element analysis of ESF near-by the person with the indication of the boundary conditions.

Taking into consideration the complicated curved shape of the surface of the human body in the protective clothing, the solution for field problem (1), (2) can be made, using the one of the currently known numerical methods [9]. In this research for the numerical solution the method of finite elements was used, which realized in the software package COMSOL Multiphysics.

As the initial data, the information about the distribution of charges on the surfaces of the insulating clothing set was used; such set was made of PVC-compound and included jacket, bib overalls, hood, respirator, gloves and shoes, made from PVC. The analysis of the experimental researches results showed that the highest density of electric charge was fixed on the outer surfaces of the insulating clothing sets, made from PVC-compound. Based on those assumptions, simulation and calculations were made for the outer layer on the brink "insulating suit - air" for two situations:

- in equal distribution of charges the maximum density of charges was $\sigma_{\mathrm{q}}=1 \cdot 10-7 \mathrm{C} / \mathrm{m}^{2}$;

- in unequal distribution of charges: the head, torso and neck- $\sigma_{\mathrm{q}}=1 \cdot 10-7 \mathrm{C} / \mathrm{m}^{2}$; the hands and legs $-\sigma_{\mathrm{q}}=$ $1 \cdot 10-10 \mathrm{C} / \mathrm{m}^{2}$.

Numerical calculation of potentials and ESF intensity demonstrated that in the equal distribution of charges the field was unequal and the maximum value of potentials was achieved in the worker's chest and neck area, as could be followed from the Figure A1 as a result of computer calculations. Lines of force of the electric field started on the surface of clothing and directed to a grounded plane of the cube, i.e. to the surface, on which the employee located (Fig. A2). The results of computer simulation provided the opportunity to get quantitative values of ESF energy indicators, taking into consideration different distribution of surface charge in time and on the surface of the protective clothes. 


\section{Discussion}

The schedule of distribution of potentials on the worker from the ground, which is considered as the point of zero potential, to the head was shown in Fig. 4.

\section{Potential, $\mathrm{kV}$}

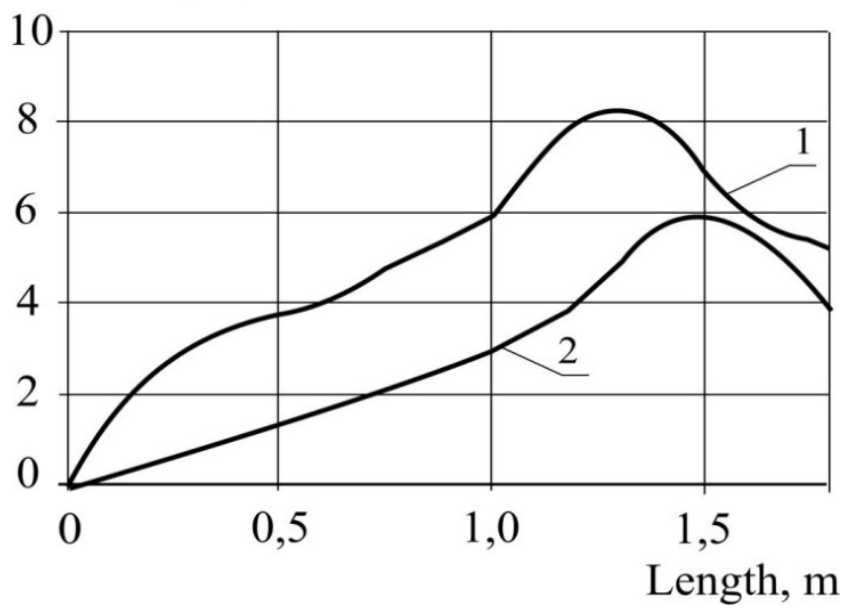

Figure 4. The distribution of potentials on the surface of protective clothing: 1 - equal distribution of charges; 2 - unequal distribution of charges.

For the maximum fixed value of the surface charge density $\sigma_{q}=10^{-7} \mathrm{C} / \mathrm{m}^{2}$ the maximum value of potential is $8 \mathrm{kV}$, which is observed on the front area of the clothing. The calculation, made for unequal distribution of surface charges, showed that increased values of potentials grouped in the areas of increased density of charges - on the front side of the clothes, on the neck and head.

The change of the ESF intensity value on the surface of the clothing is as follows (Fig. 5).

Intensity $\mathrm{E}, 10^{-4} \mathrm{~V} / \mathrm{m}$

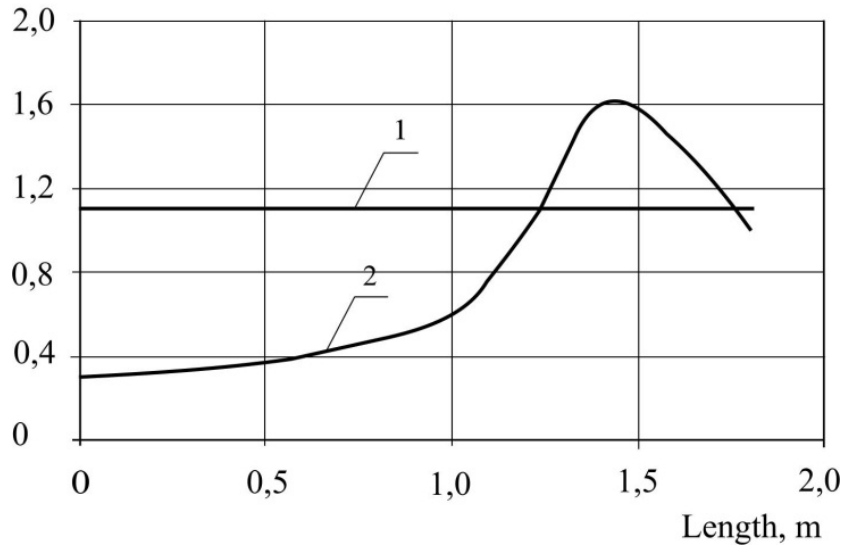

Figure 5. The distribution of intensity on the surface of protective clothing: 1 - equal distribution of charges; 2 - unequal distribution of charges.

It is known that any kind of inequality increases the ESF intension. The results of calculation confirmed this. The maximum value of intension for the unequal distribution of charges increased and was $1.6 \cdot 10^{-4} \mathrm{~V} / \mathrm{m}$. It should be noted that the value of the field intensity on the surface of clothing was far below the breakdown voltage of polymeric materials.
This means that ESF does not damage the protective elements and does not affect the reliability of products.

The growth of ESF inequality on the surface of the protective clothing set has caused the changes in the distribution of the field in the space near the worker (Fig. 6). Intensity $\mathrm{E}, 10^{-4} \mathrm{~V} / \mathrm{m}$

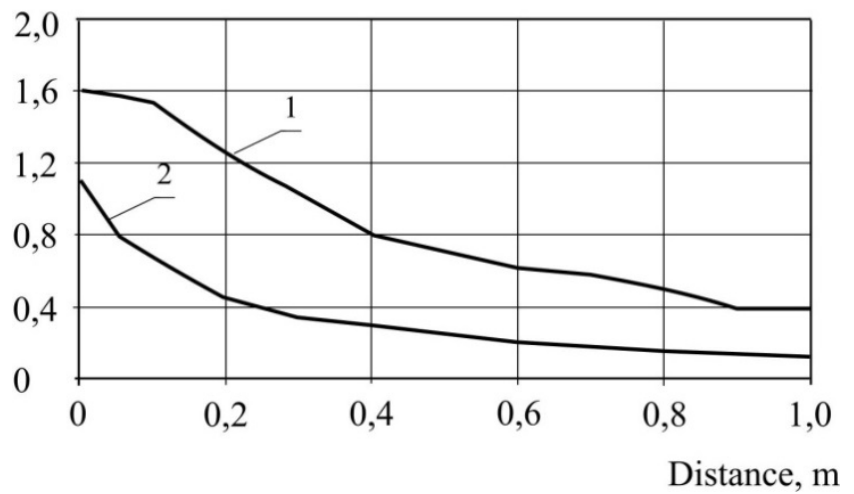

Figure 6. The distribution of electrostatic field intensity in the surrounding area near the worker: 1 - unequal distribution of the surface density of charges; 2 - equal distribution of the surface density of charges.

As we can see from Fig. 6, reduce of intension at unequal distribution of charges occurs at a slower speed compared to the equal distribution. Computer experiments have confirmed that the distribution of intension $\mathrm{E}_{\mathrm{h}}$ in the area near the worker is described by the exponential dependence, which is:

- for unequal distribution of charges

$$
E_{h}=1.6 \cdot 10^{-4} \exp (-1.24 l),
$$

where $l$ - the distance from the worker;

- for equal distribution of charges

$$
E_{h}=1.06 \cdot 10^{-4} \exp (-2.47 l) \text {. }
$$

Lines of force of ESF intension close in airspace at a distance of one meter from the worker and, accordingly, the intensity and density of the field energy reduce due to the increase of the distance from the charged object (Fig. 7).

Energy density, $\mathrm{mJ} / \mathrm{m}^{3}$

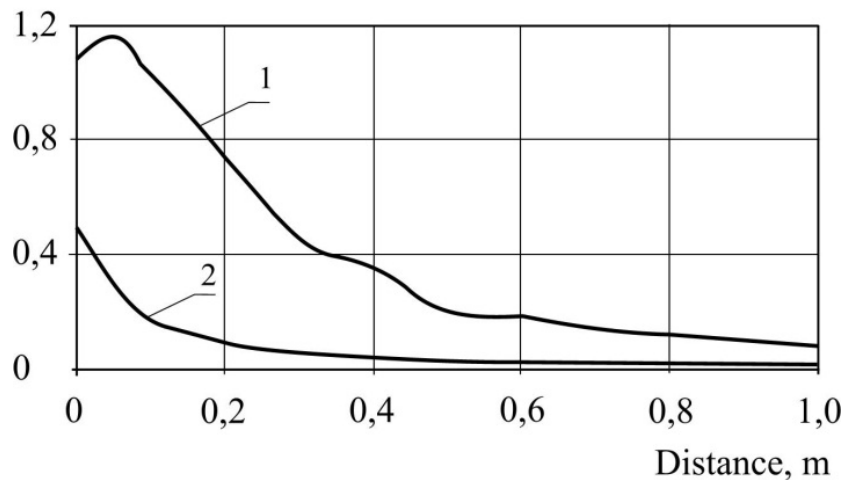

Figure 7. Distribution of energy density in the space around the worker: 1 - unequal distribution of the surface density of charges; 2 - equal distribution of the surface density of charges. 
As we can see from Fig. 7, ESF energy density on the surface of the worker's clothes during the equal distribution of charges has a maximum value $-5 \cdot 10^{-4} \mathrm{~J} / \mathrm{m}^{3}$; but during the unequal distribution of charges the increase of intension caused the increase of density of the energy, accumulated in the field, which reached a maximum value of $1.16 \cdot 10^{-3} \mathrm{~J} / \mathrm{m}^{3}$. The change of energy density $\omega_{\mathrm{e}}$ in the space near the worker approximated by such dependencies:

- for unequal distribution of charges

$$
\omega_{e}=1.14 \cdot 10^{-3} \exp (-2.82 \cdot l) ;
$$

- for equal distribution of charges

$$
\omega_{e}=0.5 \cdot 10^{-3} \exp (-3.55 \cdot l) \text {. }
$$

The decrease of energy intensity and density in $e$ times is happening at the distance of $0.3 \ldots 0.4 \mathrm{~m} \ldots$ during the equal distribution of charges and at the distance of $0.37 \ldots 0.68 \mathrm{~m}-$ during the unequal distribution of charges. Hence, it can be assumed that ESF is localized in the surface layer, thickness of which is up to $0.4 \mathrm{~m}$, during the equal distribution of charges; otherwise the distance grows up to $0.7 \mathrm{M}$.

The determination of ESF energy characteristics is necessary to evaluate the possible risks of electrostatic discharges with subsequent formation of spark with energy that exceeds the minimum ignition energy. Based on these assumptions, the conditions of security can be written as

$$
W_{d}=W_{\min } \cdot K_{s},
$$

where $\mathrm{W}_{\mathrm{d}}$ - the energy of discharge of static electricity from the surface of protective clothing, which has charges; $\mathrm{W}_{\min }-$ minimal energy for ignition of the flammable mixture; $K_{s}$ security coefficient, which is usually taken within the limits from 2.0 to 2.5 .

The evaluation of the level of energy $\mathrm{W}_{\mathrm{d}}$ is made using the results of ESF calculations. It is known that the electrical energy $\mathrm{W}_{\mathrm{e}}$, accumulated in the volume $\mathrm{V}$ with the intensity. $\boldsymbol{E}=\left\{E_{x}, E_{y}, E_{z}\right\}$, can be determined by the formula:

$$
W_{e}=0.5 \cdot \varepsilon_{0} \int_{V}\left(E_{x}^{2}+E_{y}^{2}+E_{z}^{2}\right) d V .
$$

After determining the distribution of electric potential and calculating the intensity, the numerical calculation of integral is made (4). Assuming that we determine the condition $\mathrm{W}_{\mathrm{e}}=$ $\mathrm{W}_{\mathrm{d}}$, under which the ignition of the flammable mixture cannot occur

$$
W_{\min } \geq W_{e} \cdot K_{S} .
$$

The value of the electrical energy, accumulated in the volume of $V=64 \mathrm{~m}^{3}$ in the field with the intension $\boldsymbol{E}$ according to the results of numerical integration is $W_{\mathrm{e}}=0,96$ $\mathrm{mJ}$ (for maximum charge) and $W_{\mathrm{e}}=0,28 \mathrm{~mJ}$ - for unequal distribution of charges. Check under (5) the conditions of ignition and get

$$
W_{e} \cdot K_{S}=2.4 m J
$$

For example, the minimum ignition energy $\mathrm{W}_{\min }$ of acetone is $0,406 \mathrm{~mJ}$, of sulfur aerosol $-15 \mathrm{~mJ}$. The abovementioned acquired value of the energy, accumulated in EFS, in this case will be sufficient for acetone ignition (upon the condition of the spark discharge occurrence).

\section{Conclusions}

- The method for assessment of the degree of electrification and danger of the electrostatic field allows determining a set of measures for the joint and safe functioning of the worker and technological equipment.

- When determining the suitability of protective equipment, made from polymeric materials, before the electrification and dissipation of electrical charges, must be considered not only the resistance (volume and surface), but also the value of the maximum possible charge, intensity and surface energy of electrostatic field to the appropriate working conditions.

- It was proposed the mathematical model and the method of numerical calculation of parameters of three-dimensional field near-by a man, dressed in protective clothing, made from polymeric material, at presence of surface electric charge.

\section{Appendix}

\section{The Results of Computer Simulation}

As a result of calculations, it was received the distribution of electric potential and intensity vector of ESF on the surface and near-by man in insulating clothes for maximum equal distributed surface charge density on clothing $\sigma_{\mathrm{s}}=$ $1 \cdot 10^{-7} \mathrm{C} / \mathrm{m}^{2}$ (Fig. A1, A2). Figure A2 presents the results of computer simulation of distribution of lines of force and intensity in the area.

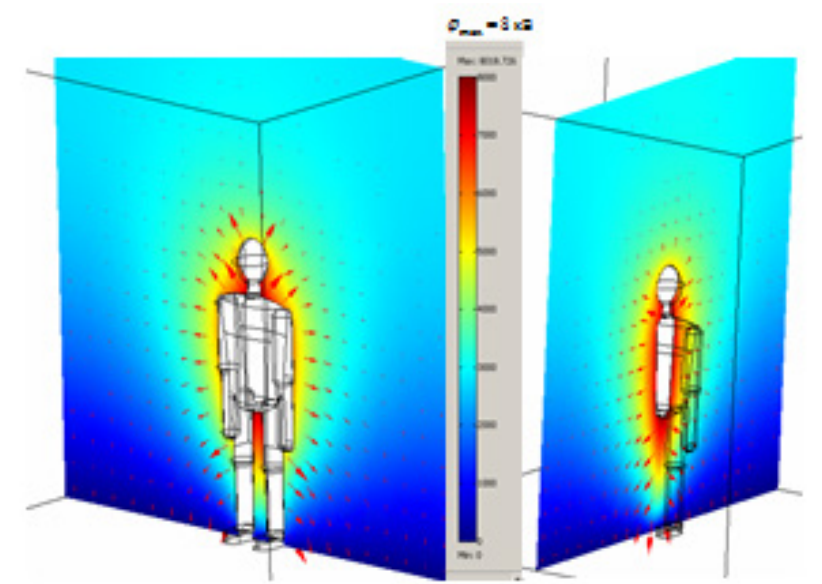

Figure A1. Distribution of electrical potential $\boldsymbol{\varphi}$ and intensity vector $\boldsymbol{E}$ (shown with arrows) in two crossings. 


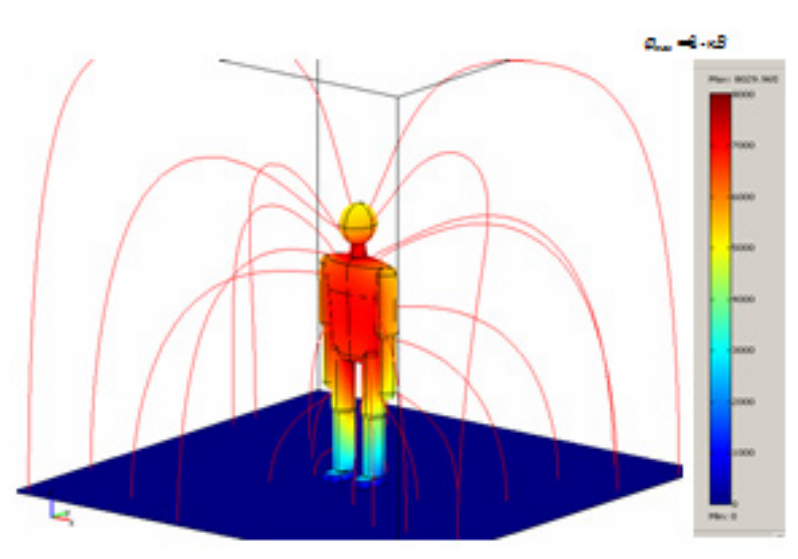

Figure A2. Distribution of electrical potential $\varphi$ on the surface of the clothing and lines of force of intensity vector $\boldsymbol{E}$ in the environment

It was designed the protective clothes (jacket and trousers) for the workers of oil refineries, made of two-layer material with PVC covering (IB). Protective clothing is put on the additional inner set, the front part of which (collar, jacket and trousers) is made using an antistatic material. Such material has special fibers, which are interwoven in the form of lattice "gridlocked" into the fabric. The stitches of the product are connected by electrically conductive threads, brand Nega-Stat. These elements create the ways for charges draining and reduce the danger of clothing ignition.

\section{REFERENCES}

[1] J. N. Chubb. Measurement of tribe and corona charging features of materials for assessment of risks from static electricity, Journal Trans. IEEE Ind. Appl, № 36 (6), 15151522, 2000.

[2] G. Luttgens, N. Wilson. Electrostatic hazards, Linacre House, Oxford, 1997.

[3] J. Paasi, L. Fast, T. Kalliohaka Risks of damage to electronics with reference o charged clothing. Journal of Electrostatics, No 63, 603-608, 2005.

[4] P. Kathirgamanathan, M. J. Toohey, J. Haase, P. Holdstock, J. Laperre, G. Schmeer-Lioe. Measurements of incendivity of electrostatic discharges from textiles used in personal protective clothing Juornal Electrostatics, № 49, 51-70, 2000.

[5] Sanitary norms of permissible intensity electrostatic field. $\mathrm{CH}$ № 1757, Kiev, 2008. [in Ukrainian].

[6] ДСТУ ЕN 1149-1. Protective clothing. Electrostatic properties. Part 1. Surface resistance (measurement method and requirements), Kiev, 2005. [in Ukrainian].

[7] L. Tretiakova. Problems methodology for assessing the electrostatic properties of plant protection personnel, Journal of Industrial electricity and electrical engineering, № 3, 3743, 2010. [in Ukrainian].

[8] L. Tretiakova, A. Seliverstov. The newest solution of individual protection for employees of nuclear power plants, Osnova, Kiev, 2016. [in Ukrainian].

[9] A. Podoltsev. Elements of the theory and numerical calculation of electromagnetic processes in conducting media, Institute of Electrodynamics, Kiev, 1999. [in Russian]. 